\title{
Observational tests of the Electro-Magnetic Black Hole Theory in Gamma Ray Bursts
}

\author{
Remo Ruffini ${ }^{1}$ \\ ICRA - International Center for Relativistic Astrophysics and Physics Department, \\ University of Rome "La Sapienza", I-00185 Rome, Italy.
}

\begin{abstract}
The Relative Space-Time Transformation (RSTT) Paradigm [44 and the Interpretation of the Burst Structure (IBS) Paradigm 45 are applied to the analysis of the structure of the burst and afterglow of Gamma-Ray Bursts within the theory based on the vacuum polarization process occurring in an Electro-Magnetic Black Hole, the EMBH theory. This framework is applied to the study of the GRB 991216 which is used as a prototype. The GRB-Supernova Time Sequence (GSTS) Paradigm, which introduces the concept of induced gravitational collapse in the Supernovae-GRB association [46], is announced and will be applied, within the EMBH theory, to GRB 980425 as a prototype in a forthcoming paper.
\end{abstract}

\section{Introduction}

I am very pleased to present here in Munich some observational tests of our Electro-Magnetic-Black-Hole theory, for short the EMBH theory, explaining some features of Gamma Ray Bursts, for short GRBs. The EMBH theory is rooted in discussions I had from 1971 to 1975 with Werner Heisenberg here in Munich, in Washington and Stanford.

GRBs are today promoting one of the most ample scientific effort in the entire field of science, both in the observational and theoretical domains. Following the discovery of the GRBs by the Vela satellites [58], the observations from the Compton satellite and BATSE had shown the isotropical distribution of the GRBs strongly suggesting a cosmological nature for their origin. It was still trough the data of BATSE that the existence of two families of bursts, the "short bursts" and the "long bursts" was presented, opening an intense scientific dialogue on their origin still active today, as we see in the talk of M. Schmidt in these proceedings.

An enormous momentum was gained in this field by the discovery of the afterglow phenomena by the BeppoSAX satellite and the optical identification of the GRBs sources at cosmological distances (see e.g. [9]). It has become apparent that fluxes of $10^{54} \mathrm{ergs} / \mathrm{s}$ are reached: during its peak emission the energy of a single GRB equals the energy emitted by all the stars of the Universe (see e.g. [18).

From an observational point of view, an unprecedented campaign of observations is at work using the largest deployment of observational techniques from space with the satellites CGRO - BATSE, Beppo-SAX, Chandra, R-XTE, XMMNewton, HETE-2, as well as the HST, and from the ground with optical KECK 

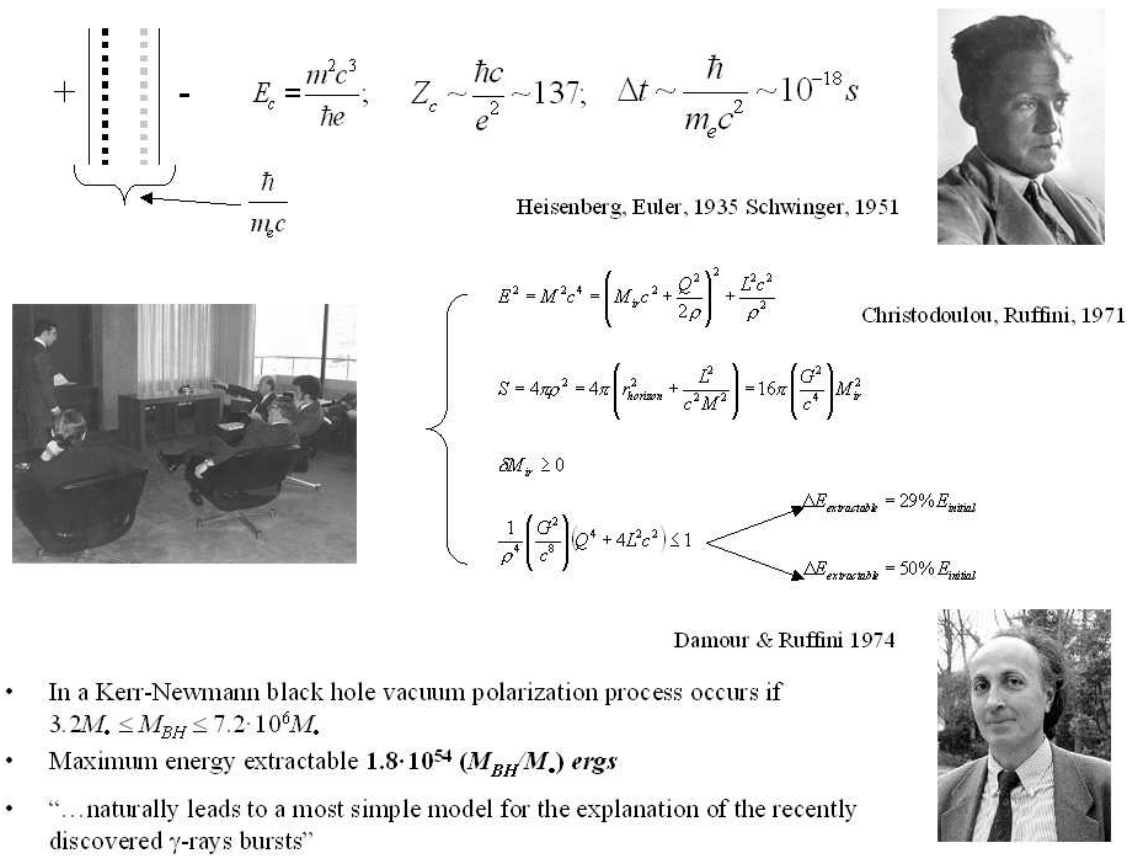

Fig. 1. Theoretical background of the EMBH model. The critical field against the breakdown of the vacuum is given next to a picture of Werner Heisenberg. The mass formula of EMBH is given next to the picture of the Ph.D. thesis discussion in Princeton by Demetrios Christodoulou, at the age of 19, in front of the committee with members: R. Ruffini (supervisor), J.A. Wheeler, E. Wigner and D. Wilkinson. The energetics of the vacuum polarization process of an EMBH is given next to the picture of T. Damour. These results were obtained, with R. Ruffini as supervisor, at Princeton University in 1974 during the preparation of the thesis of Doctorat d' Etat finally presented in Paris.

and the VLT and radio by the VLA observatories. Possibility of further examining correlation with detection of ultrahigh energy cosmic rays and neutrinos should be reachable in the near future thanks to developments of the AUGER and AMANDA experiments (see also [21]).

From a theoretical point of view, the GRBs offers comparable opportunities to develop new domains in yet untested directions of fundamental science. For the first time within the EMBH model, see Fig. 2, the opportunity exist to theoretically approach the following fundamental issues:

1. extreme relativistic hydrodynamic phenomena of an electron positron plasma expanding with sharply varying Lorentz gamma factors in the range $10^{2}$ to $10^{4}$. Analyze as well the very high collision of such expanding plasma with baryonic matter reaching intensities $10^{40}$ larger then the ones usually obtained in Earth based accelerators, see [50] and references therein, 


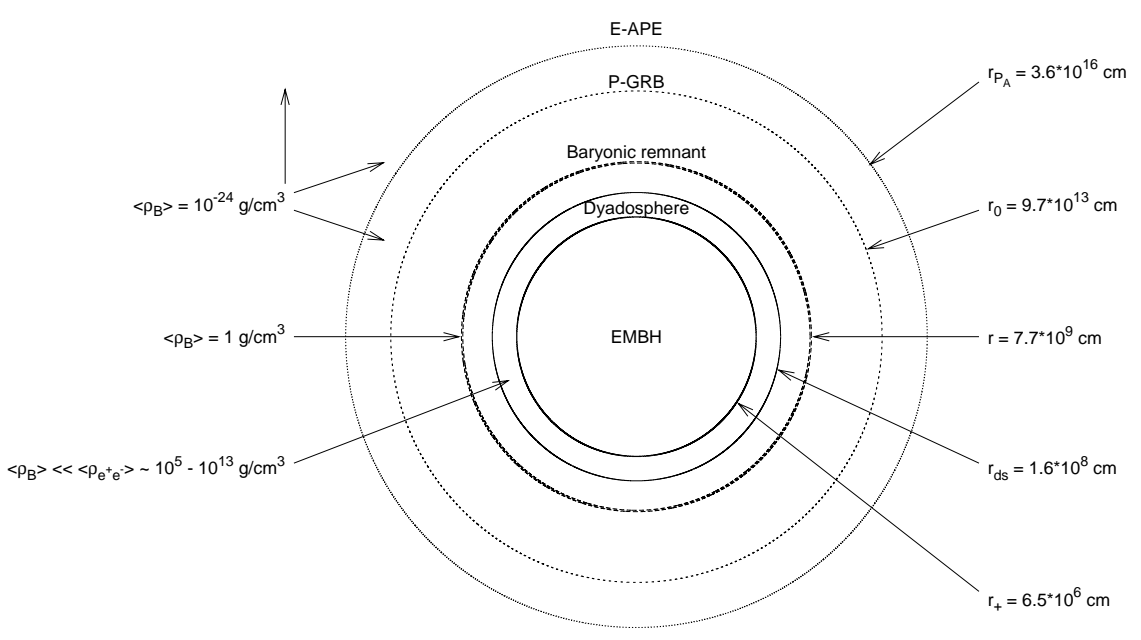

Fig. 2. Selected events of the expansion of the pulse in the EMBH model are represented, together with the distance from the EMBH in the laboratory frame at which they occur and with the density of the surrounding medium. The radial distances are represented in logarithmic scale.

2. the bulk process of vacuum polarization created by overcritical electromagnetic fields, in the sense of Heisenberg, Euler [22 and Schwinger [57]. This long sought quantum ultrarelativistic effect, not yet convincingly observed in heavy ion collision on the Earth in Darmstadt, may indeed make its first appearance in the strong electromagnetic fields developed in astrophysical conditions during the process of gravitational collapse to a black hole, see 36. 41, 42,

3. a novel form of energy source: the extractable energy of a black hole, introduced by Christodoulou and Ruffini [7]. The enormous energies, released almost instantly in the observed GRBs, points to the possibility that for the first time we are witnessing the release of the extractable energy of an $\mathrm{EMBH}$, during the process of gravitational collapse itself. We can compute and, if observationally confirmed, have the opportunity to study all general relativistic effects as the horizon of the Black hole is approached and is being formed, together with the associated ultrahigh energy quantum phenomena, see [6, [4, 53].

It is clear that in the approach to such a vast new field of research implying previously unobserved relativistic regimes it is not possible to proceed as usual adopting an uncritical comparison of observational data to theoretical models within the classical schemes of astronomy and astrophysics. Some insight to the new approach needed can be gained from past experiences in the interpretation of relativistic effects in high energy particle physics as well as from the explanation of some relativistic effects observed in the astrophysical domain. All those 
relativistic regimes are however much less extreme then the new ones encountered in GRBs.

There are at least three major new features in relativistic systems which have to be taken into due account:

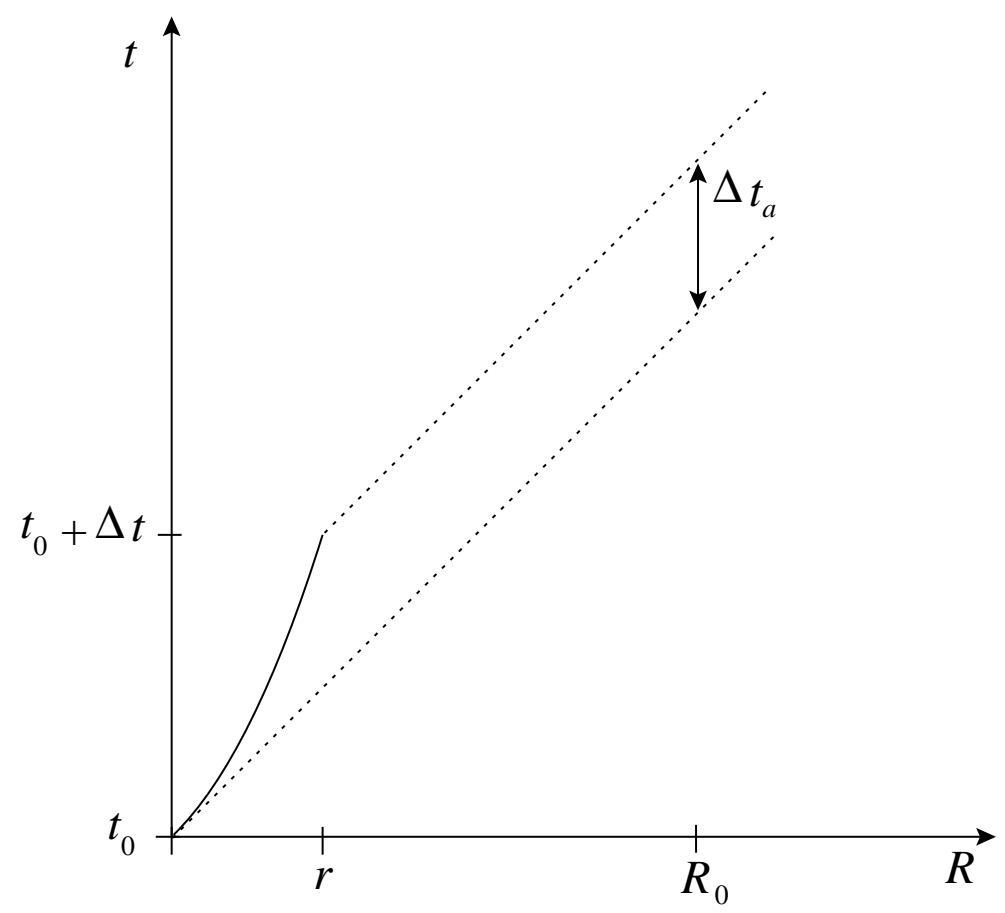

Fig. 3. This qualitative diagram illustrates the relation between the laboratory time interval $\Delta t$ and the arrival time interval $\Delta t_{a}$ for a pulse moving with velocity $v$ in the laboratory time (solid line). We have indicated here the case where the motion of the source has a nonzero acceleration. The arrival time is measured using light signals emitted by the pulse (dotted lines). $R_{0}$ is the distance of the observer from the EMBH, $t_{0}$ is the laboratory time corresponding to the onset of the gravitational collapse, and $r$ is the radius of the expanding pulse at a time $t=t_{0}+\Delta t$. See also [44.

1. Practically the totally of data in astronomical and astrophysical systems are acquired by using photons arrival time. It was Einstein [15 at the very initial steps of special relativity to caution on the use of such an arrival time analysis and to state that, when dealing with objects in motion, due care should be put in defining time synchronization procedure in order to construct the correct spacetime coordinate grid (see Fig. 3). It is not surprising that as soon as the first bulk motions relativistic effects were observed by radio and optical telescopes their interpretations within the classical framework of 
astrophysics led to the concept of "superluminal" motion. These observations refer to extragalactic radio sources, with Lorentz gamma factors $\sim 6$ [ [ ] and to microquasars in our own galaxy with Lorentz gamma factor $\sim 5$ [28]. It has been recognized [38] that no "superluminal" motion exist as soon as the prescriptions indicated by Einstein are used in order to establish the correct spacetime grid for the astrophysics systems. In the present context of GRBs, where the Lorentz gamma factor can easily surpass $10^{2}$, the direct application of classical concepts leads to enormous "superluminal" behaviors 50. An approach based on classical arrival time considerations, as done sometime in current literature, completely subvert the causal relation in the observed astrophysical phenomenon.

2. One of the clear success of relativistic field theorists has been the understandings of the role of four momentum energy conservation laws in multiparticle collisions and decays such as the reaction: $n \rightarrow p+e+\bar{\nu}$. From the works of Pauli anf Fermi it became clear how in such processes, contrary to the case of classical mechanics, it is impossible to analyze a single term of the decay, the electron or the proton or the neutrino or the neutron, out of the context of the global point of view of the relativistic conservation of the total four momentum of the system, which involves the knowledge of the system during the entire decay process. These rules are routinely used by workers in high energy particle physics and have become part of their cultural background. If we apply these same rules to the case of the relativistic system of a GRB it is clear that it is just impossible to consider a part of the system, e.g. the afterglow, out of the general conservation laws and history of the entire relativistic regime of the system. The description of the sole afterglow, as has been done at times in the literature, could indeed be done within the framework of classical astronomy and astrophysics, but not in a relativistic astrophysics where the entire space-time grid necessary for the description of the afterglow depends on all the previous relativistic part of the worldline of the system.

3. The very lifetime of a phenomenon has not an absolute meaning, special and general relativity have shown. It depends both from inertial reference frame of the laboratory and of the observer and their relative motion. Such a phenomenon, generally expressed in the "twin paradox" has been extensively checked and confirmed to extreme high accuracy in elementary particle physics in the CERN experiments. This situation is much more extreme in GRBs due to the very large (in the range $10^{2}-10^{4}$ ) and time varying (on time scales ranging from fractions of seconds to months) gamma Lorentz factors between the Laboratory frame and the far away observer. Such an observer is moreover in the GRBs context further affected by the cosmological recession velocities of its local Lorentz frame.

These are some of the reasons why we have recently presented a basic Relative Space-Time Transformations RSTT paradigm [44] to be applied prior to the interpretation of GRBs data. 

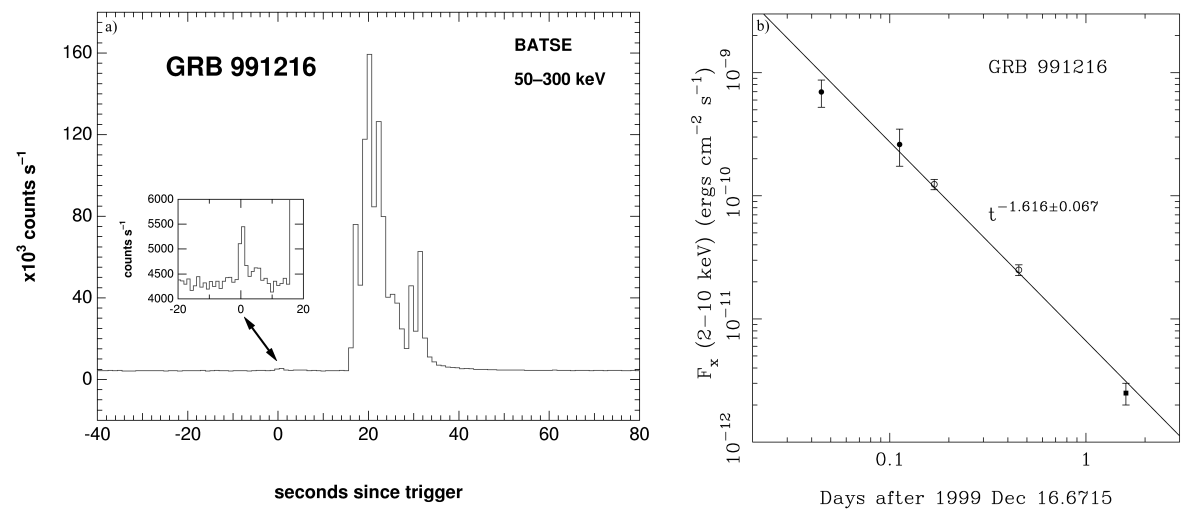

Fig. 4. a) The peak emission of GRB 991216 as seen by BATSE (reproduced from [1]); b) The afterglow emission of GRB 991216 as seen by XTE and Chandra (reproduced from 20]).

The first step is the establishment of the constitutive equations relating:

a) The comoving time of the pulse $(\tau)$

b) The laboratory time $(t)$

c) The arrival time $\left(t_{a}\right)$

d) The arrival time at the detector $\left(t_{a}^{d}\right)$

The book-keeping of the four different times and corresponding space variables must be done carefully in order to keep the correct causal relation in the time sequence of the events involved.

The RSST paradigm reads: "the necessary condition in order to interpret the GRB data is the knowledge of the entire worldline of the source from the moment of gravitational collapse. In order to meet this condition, given a proper theoretical description and the correct constitutive equations, it is sufficient to know the energy of the dyadosphere and the mass of the remnant of the progenitor star".

Clearly such an approach is at variance with the works in the current literature which mainly emphasize either some qualitative description of the sources or some quantitative phenomenological multiparameter fitting of the sole afterglow era.

- Many works in the literature have addressed the issue of the sources of GRBs. They include works on binary neutron stars mergers (see e.g. 114,29, 24, 250, , black hole - white dwarf [18] and black hole - neutron star binaries [30.27, Hypernovae (see [32]), failed supernovae or collapsars (see [63,23]), supranovae (see [60,61]). Only those based on binary neutron stars have reached the definition of detailed quantitative estimates of a model, but they present serious difficulties in the energetics, as well as in the explanation of "long bursts" (see [54,62]), and in the observed location of the GRBs' sources in star forming regions (see [5). In the remaining cases was presented a sole 
qualitative analysis of the sources without addressing the overall problem from the source to the observations: the necessary details to formulate the equations of the dynamical evolution of the system are generally still missing.

Other works in the literature have mainly addressed the problem of fitting the data of the afterglow observations by phenomenological analysis. They are separated in two major classes:

- The "internal shock models", first introduced by [39], are by far the most popular ones having been developed in many different aspects, e.g. by [31,55, 16, 17. The underlying assumption is that all the variabilities of GRBs in the range $\Delta t \sim 1 \mathrm{~ms}$ up to the overall duration $T$ of the order of $50 \mathrm{~s}$ are determined by a yet undetermined "inner engine". The difficulties of explaining the long time scale bursts by a single explosive event has evolved into a variety of assumptions on yet unspecified family of "inner engines" with a prolonged activity (see e.g. [34] and references therein).

- The "external shock models", also introduced by 26], are less popular today. There is the distinct possibility, within these models, that "GRBs' light curves are tomographic images of the density distribution of the medium surrounding the sources of GRBs" ([12], see also [11,13 and references therein). It is generally outlined that the structure of the burst does not depend directly from the "inner engine".

All these works encounter the above mentioned difficulties, they present only a piecewise description of the GRB phenomenon and by neglecting the earlier phases, their space time grid is undefined and, as we have shown in detail in [50], results are reached at variance from the ones obtained in a complete and unitary description of the GRBs phenomenon. We outline in the following how such an unitary description naturally leads to new characteristic features both in the burst and afterglow of GRBs.

In a series of papers, we have developed the above mentioned EMBH model 40 which has the great advantage, despite its simplicity, that all eras of the model, following the process of gravitational collapse, are described by precise field equations which can then be numerically integrated. The three basic starting points are:

- the extractable energy of an EMBH introduced in [7],

- the vacuum polarization process à la Heisenberg-Euler-Schwinger 22,57 in the field of an EMBH, first computed in [10],

- the fact that vacuum polarization process can indeed be a realization of the reversible transformation of an EMBH introduced in [7].

These were the themes of discussions with Werner Heisenberg. He was supposed to inaugurate the 1975 Varenna Summer School [19] directed to these subjects if he had not died a few weeks earlier. In that school and in 10 the possibility that the process in 22,57 duly extended to the EMBH were at the very basis of the explanation of GRBs was advanced (see Fig. 1). 

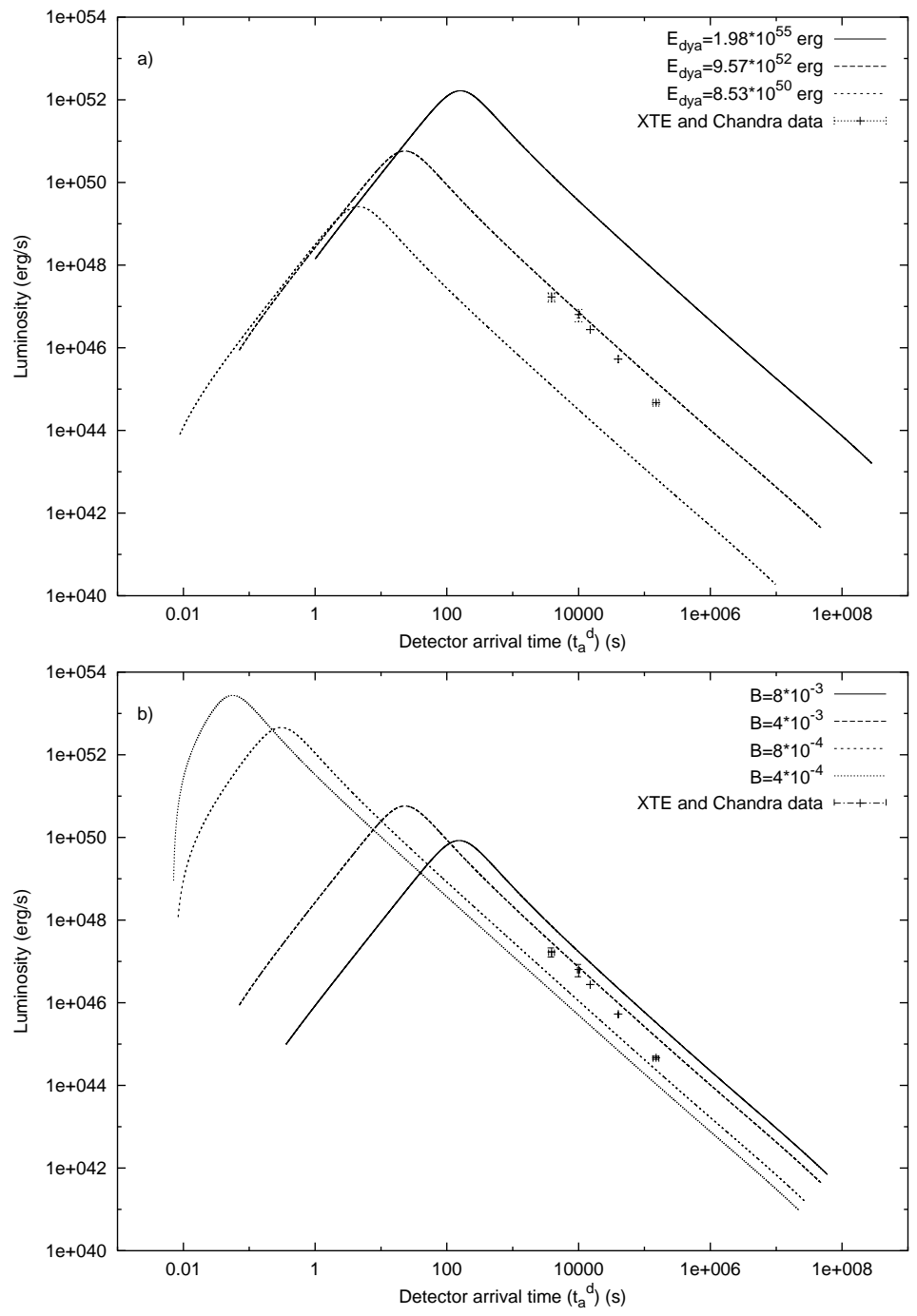

Fig. 5. a) Afterglow luminosity computed for an EMBH of $E_{d y a}=8.53 \times 10^{50} \mathrm{ergs}$, $E_{d y a}=9.57 \times 10^{52} \mathrm{ergs}, E_{d y a}=1.98 \times 10^{55} \mathrm{ergs}$ and $B=4 \times 10^{-3}$. b) for the $E_{d y a}=9.57 \times 10^{52}$, we give the afterglow luminosities corresponding respectively to $B=4 \times 10^{-4}, 8 \times 10^{-4}, 4 \times 10^{-3}, 8 \times 10^{-3}$. 
Following the Beppo SAX observations and the energetics requirements, we have returned to our EMBH model [40] and developed the dyadosphere concept [36. The dynamics of the $e^{+} e^{-}$-pairs and electromagnetic radiation of the plasma generated in the dyadosphere propagating away from the EMBH in a sharp PairsElectro-Magnetic pulse (the PEM pulse) has been studied by us and validated by the numerical codes at Livermore Lab [41]. The collision of the still optically thick PEM pulse with the baryonic matter of the remnant of the progenitor star has been again studied and validated by the Livermore Lab codes 42]. The further evolution of the sharp pulse of pairs-electromagnetic radiation and baryons (the PEMB pulse) further proceeds with increasing values of the Lorentz gamma factor until the condition of transparency is reached [2]. At this stage the Proper-Gamma Ray Burst (P-GRB) is emitted [45] and a pulse of AcceleratedBaryonic-Matter the (ABM pulse) is injected in the interstellar medium giving rise to the afterglow.

The interaction of the ABM-Pulse giving origin to the afterglow has been recently developed and presented in detail in 50. We recall the minimum set of assumptions we have adopted:

1. the collision of the ABM pulse is assumed to occur with a constant homogeneous interstellar medium of number density $n_{\text {ism }} \sim 1 \mathrm{~cm}^{-3}$. The energy emitted in the collision is assumed to be instantaneously radiated away (fully radiative condition). The description of the collision and emission process is done in spherical symmetry, taking only the radial approximation neglecting all the delay emission by scattered radiation.

2. special attention is given to numerically compute the power of the afterglow as a function of the arrival time using the correct constitutive equations for the space-time transformations in line with the RSTT paradigm.

3 . finally some approximate solutions are adopted in order to obtain the determination of the power law indexes of the afterglow flux and compare and contrast them with the observational results as well as with the alternative results in the literature.

In [50] we have considered uniquely the above radial approximation in order to concentrate on the special role of the correct space time transformations in the RSST paradigm and to explicitly illustrate their impact on the determination of the power law index of the afterglow. This topic has been unduly neglected in the literature. We enter in a forthcoming papers both in the details of the role of beaming of the radiation and of the diffusion due to off axis emission [51,3].

It is now clear after the observations of GRB 980425 that the afterglow phenomena can present, especially in the optical and radio wavelengths, features originating from phenomena spatially and causally distinct from the GRB phenomena. There is evidence in four different GRB systems, including GRB 991216 due to the observed emission in the iron lines and their shift, that a second component exist associated to the GRBs: a supernova. This supernova explosion follows in time the GRB emission and occurs at distances of $10^{16}-10^{17} \mathrm{~cm}$ away from the location where the gravitotional collapse to the EMBH has occurred. The space time analysis of these events can be correctly performed when 


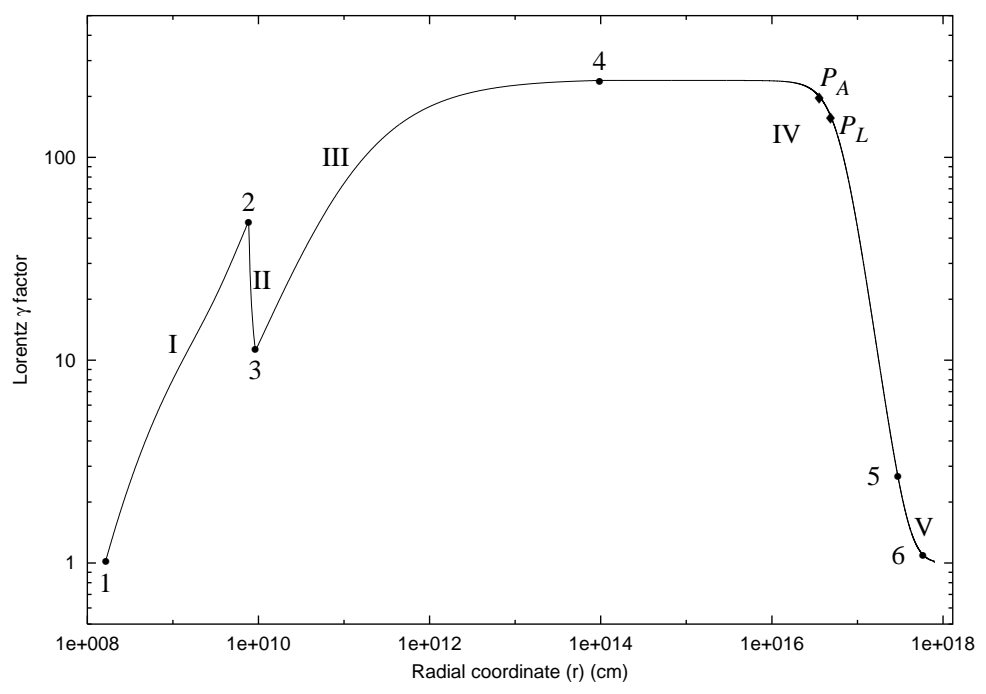

Fig. 6. The theoretically computed Lorentz gamma factor for the parameter values $E_{\text {dya }}=9.57 \times 10^{52} \mathrm{erg}, B=4 \times 10^{-3}$ is given as a function of the radial coordinate in the laboratory frame. The corresponding values in the comoving time, laboratory time and arrival time are given in 50]. The different eras, indicated by roman numerals, are illustrated in the text, while the points $1,2,3,4,5$ mark the beginning and end of each of these eras. The points $P_{L}$ and $P_{A}$ mark the maximum of the afterglow flux, respectively in emission time and in arrival time (see 445). The point 6 is the beginning of Phase $\mathrm{D}$ in era $\mathrm{V}$. At point 4 the transparency condition is reached.

the RSTT paradigm is adopted 44,45]. These facts have motivated us to introduce the novel concept of induced supernovae 46 . In the current litterature the existence of these two different components has not been recognized and attempts have been made of fitting the data of the supernova, in the x-rays as well as in the optical and radio, as part of the afterglow within the framework of a multiparameters fitt. For the above mentioned reasons, such an approach adds further difficulties to the already critical situation of the current literature.

We have therefore confronted the theoretical results of the EMBH model with the data of GRB 991216 as a prototypical case (see Fig. (4). The reason of this choice are simply given:

1. This is one of the strongest GRBs in x-rays and is also quite general in the sense that shows relevant cosmological effects. It radiates mainly in X-rays and in $\gamma$-rays and less then 3\% is emitted in optical and radio bands (see 20]). Also the emission of the supernova, inferred from the iron lines, is in this case weaker then the autentic GRB energy flux.

2. The excellent data obtained by BATSE on the burst [1] are complemented by the data on the afterglow acquired by the Chandra [35] and RXTE [8], 
and also superb data have been obtained from spectroscopy of the iron lines 35 .

3. A very precise value for the slope of the energy emission during the afterglow as a function of time has been obtained: $n=-1.64$ [59] and $n=-1.616 \pm$ 0.067 20].

The comparison of the EMBH model to the data of the GRB 991216 and its afterglow has naturally led to a new paradigm for the interpretation of the burst structures (IBS paradigm) of GRBs 45]. The IBS paradigm reads: "in GRBs we can distinguish an injector phase and a beam-target phase. The injector phase includes the process of gravitational collapse, the formation of the dyadosphere, as well as era I (the PEM pulse), era II (the engulfment of the baryonic matter of the remnant) and era III (the PEMB pulse). The injector phase terminates with the P-GRB emission. The beam-target phase addresses the interaction of the $A B M$ pulse, namely the beam generated during the injection phase, with the ISM as the target. It gives rise to the E-APE and the decaying part of the afterglow".

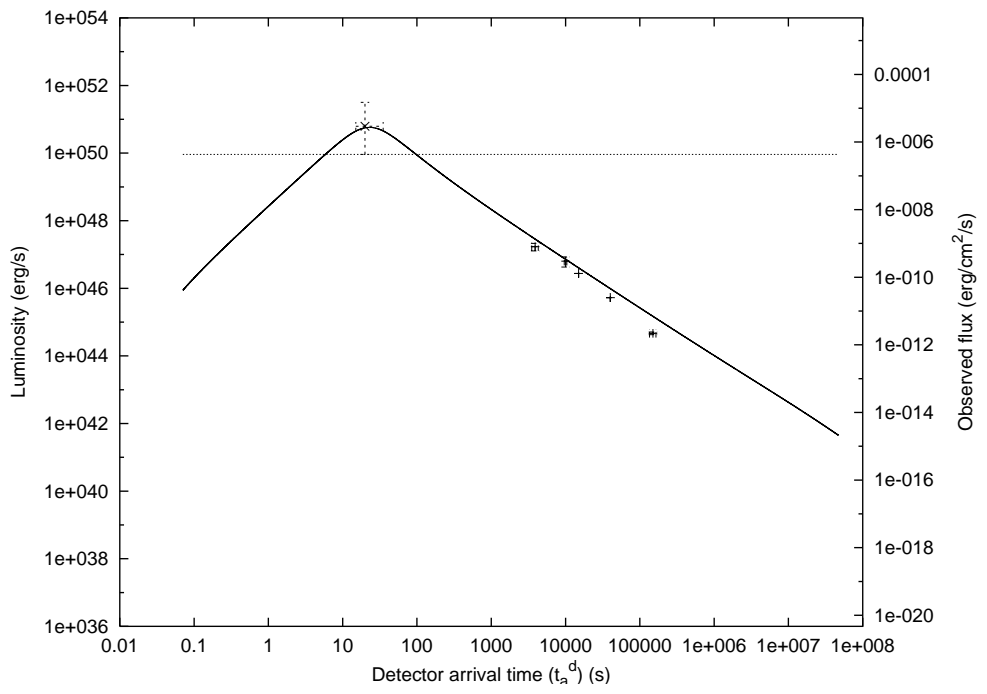

Fig. 7. Best fit of the afterglow data of Chandra, RXTE as well as of the range of variability of the BATSE data on the major burst, by a unique afterglow curve leading to the parameter values $E_{d y a}=9.57 \times 10^{52} \mathrm{erg}, B=4 \times 10^{-3}$. The horizontal dotted line indicates the BATSE noise threshold. On the left axis the luminosity is given in units of the energy emitted at the source, while the right axis gives the flux as received by the detectors.

We recall that:

a) The injector phase starts from the moment of gravitational collapse all the way to the emission of the proper GRB (the P-GRB) and encompasses the following eras: 
- The zeroth era: the formation of the dyadosphere;

- The era I: the expansion of the PEM pulse;

- The era II: the interaction of the PEM pulse with the remnant left over by the collapse of the progenitor star;

- The era III: the further expansion of the PEMB pulse; The injector phase is concluded by the emission of the P-GRB and the ABM pulse, as the condition of transparency is reached.

b) The beam-target phase, in which the accelerated baryonic matter (ABM) generated in the injector phase collides with the interstellar medium (ISM), gives origin to the afterglow and encompasses the following eras:

- The Era IV: the ultra relativistic and relativistic regimes in the afterglow: the emitted flux first increases to reach a maximum value and then monotonically decrease following well defined power laws in the arrival time;

- The Era V: the approach to the non relativistic regimes in the afterglow, also describable by specific power laws in the arrival time;

Some qualitative representation of these eras as a function of the radial coordinate, in logarithmic scale are represented in Fig. 2 .

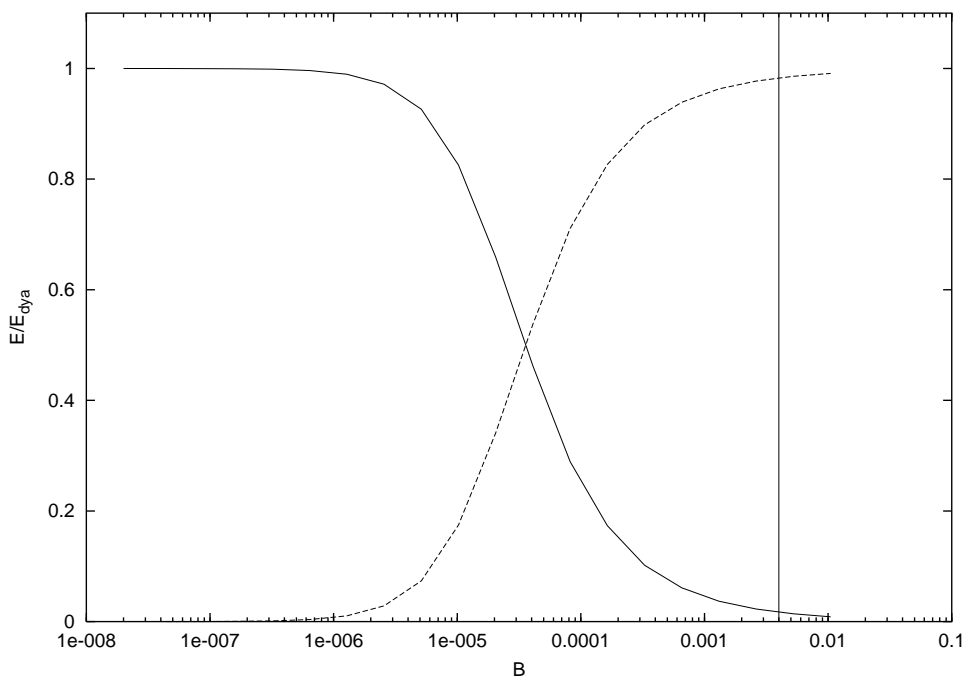

Fig. 8. Relative intensities of the E-APE (dashed line) and the P-GRB (solid line), as predicted by the EMBH model corresponding to the values of the parameters determined in Fig. 7, as a function of $B$. Details are given in [50]. The vertical line corresponds to the value $B=4 \times 10^{-3}$.

The comparison of the EMBH theory to the data of GRB 991216 has allowed the determination of the only two free parameters of the theory: the energy of 
the Dyadosphere $E_{d y a}$ and the mass of the baryonic remnant left over by the collapse of the progenitor star, measured in units of the $E_{d y a}$, defined by the dimensionless $B$ parameter. Details are given in 50 .

We have then obtained, for the first time, the complete history of the Lorentz gamma factor from the moment of gravitational collapse to the latest phases of the afterglow observations (see Fig. 6). We have as well determined the entire space time grid of the GRB 991216 by giving (see Table 1 in [50]) the radial coordinate of the GRBs phenomenon as a function of the four coordinate time variables. The extreme relativistic regimes at work in GRB 991216 lead to enormous superluminal behavior (up to $10^{4} c$ !) if the classical astrophysical concepts were adopted using the arrival time as the independent variable (see Table 1 in [50). In turn this implies that any causal relation based on classical astrophysics and the arrival time data, as often done in current GRBs literature, is incorrect.

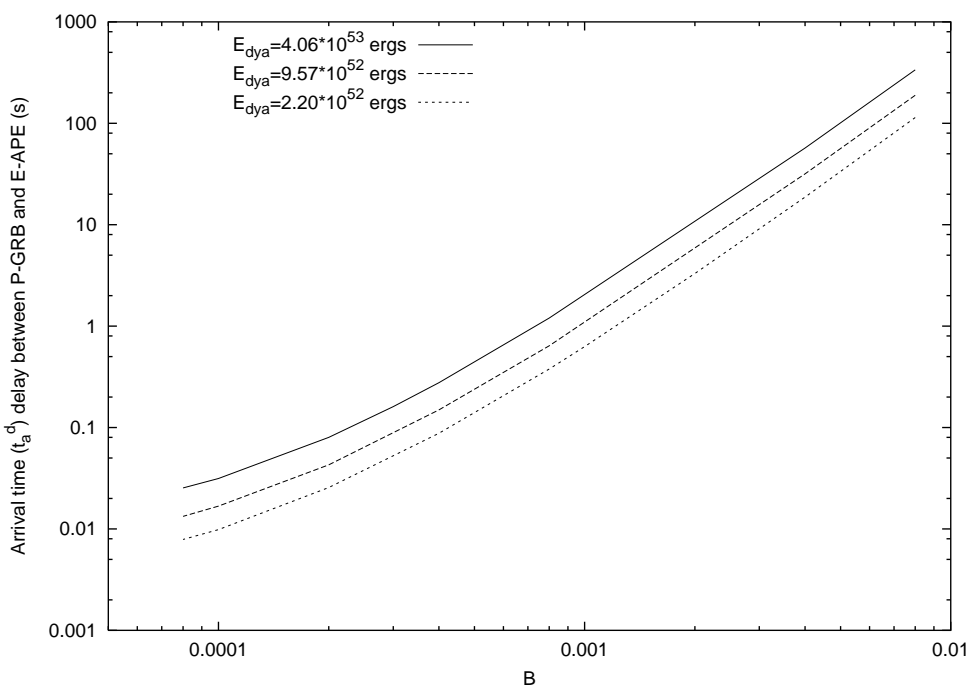

Fig. 9. Time delays between P-GRB and E-APE as a function of the B parameter for selected values of the $E_{d y a}$.

We have just stressed how the analysis of the sole afterglow of GRB 991216 data, obtained by BATSE and the Chandra and RXTE satellites, has allowed to fix the only two free parameters of the EMBH theory. As a first byproduct of this analysis we can conclude, at variance with results in 20,33 pointing to a sharply collimated beamed emission in GRB 991216, that no evidence of beaming is found as a consequence of the perfect agreement between the observed slope of the afterglow and the theoretical value obtained within the EMBH model.

We can now proceed to acquire the predictions of the EMBH theory with reference to two fundamental quantities and their role within the GRBs structure: the P-GRB and the peak emission of the afterglow. 

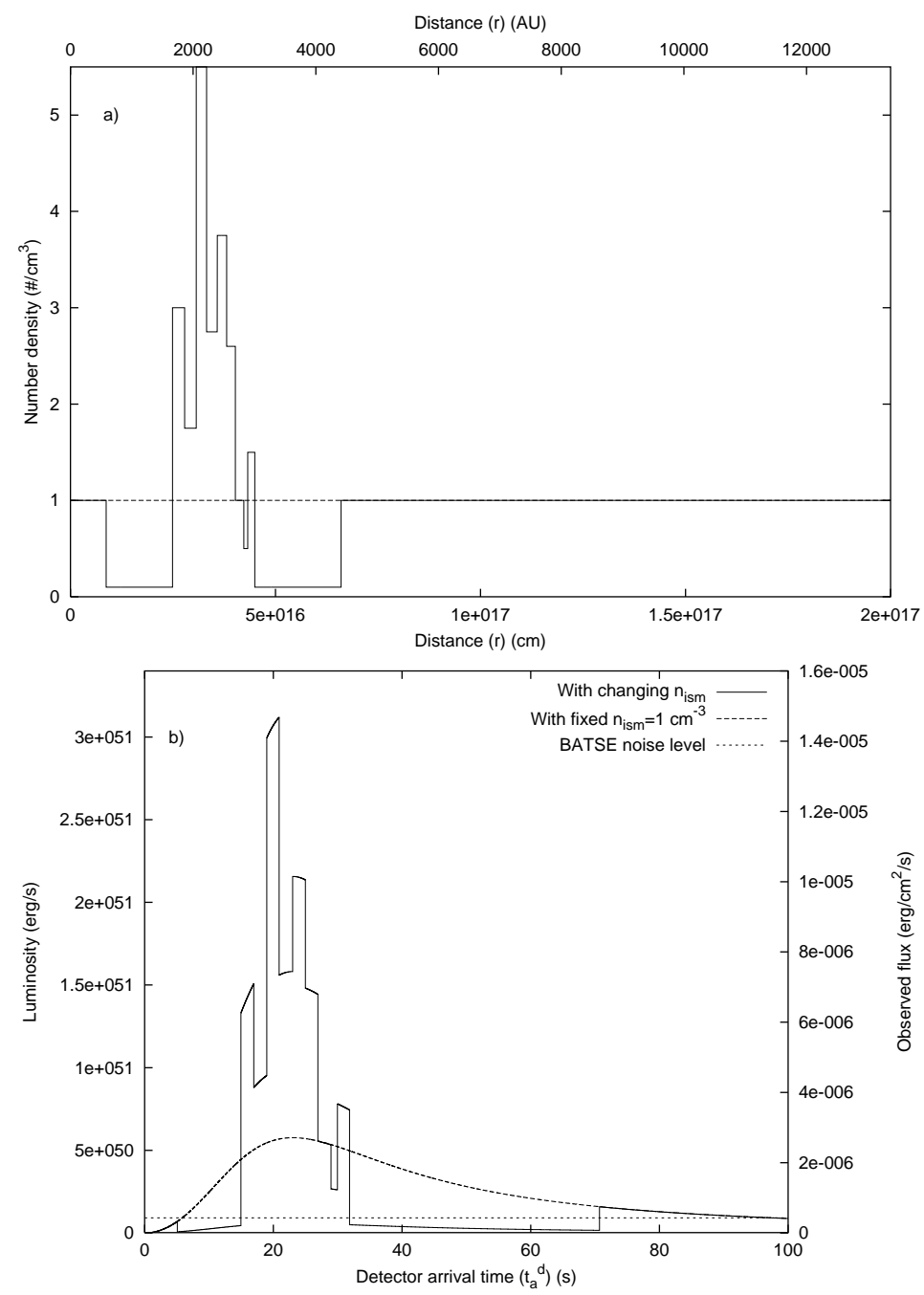

Fig. 10. a) The density contrast of the ISM cloud profile introduced in order to fit the observation of the burst of GRB 991216. The dashed line indicates the average uniform density $n=1 \mathrm{~cm}^{-3}$. b) Flux computed in the collision of the ABM pulse with an ISM cloud with the density profile given in a). The dashed line indicates the emission from an uniform ISM with $n=1 \mathrm{~cm}^{-3}$. The dotted line indicates the BATSE noise level. Note that 20 seconds in arrival time do corresponds to $\sim 5.0 \times 10^{16} \mathrm{~cm}$ in laboratory frame! Details are given in Table 1 of [50]. Compare and contrast these theoretical curves with the actual data reported in Fig. 4. 
It soon appeared clear that the :

1. the so called "long bursts" observed by BATSE are actually not bursts at all. Once the proper space-time greed is given it is immediately clear that the long bursts are generated at distances of $4 \times 10^{16} \mathrm{~cm}$ from the EMBH. The long burst coincides with the Extended-Afterglow-Peak-Emission, which we will call E-APE, of the afterglow: they were interpreted as bursts only due to the high threshold of the BATSE detectors see Fig. 7 .

2. The time variability observed in simply due to the density inhomogeneities intrinsic in an interstellar cloud, as the ABM pulse impact on it 47, also see Fig. 10.

3. The previous two conclusions are based on the simplified pure radial description of the afterglow presented in [50]. The effects of angular scattering and spreading in the signal has been considered [51,3]. This more complex approach leads to interesting new results, but does not affect the two above conclusions.

The hunt of the P-GRB then started. The interest in identifying it is mainly because some general relativistic and relativistic quantum field theory effects originating in the process of gravitational collapse during the formation of the EMBH are, in principle, encoded in the structure of the P-GRB.

Having the only two free parameter of the EMBH theory been fixed, there are two fundamental diagrams to be analyzed. The first, Fig. 9 relates the precise separation in time between the E-APE and the P-GRB, as a function of the amount of baryonic matter left over by the gravitational collapse of the progenitor star expressed by parameter $B$ and for selected values of the $E_{d y a}$. The second relates the intensities of the P-GRB to the E-APE, in units of the $E_{d y a}$, to the parameter $B$, see Fig. 8. We stress that indeed this last diagram is an universal one, in the adopted variables. From these diagrams we can identify with the precision of a few percent in the intensity and with an approximation of a few tenth of milliseconds the P-GRB with the "precursor" in the BATSE data, (see Fig. (1 and Fig. 11).

Before concluding we would like to stress one final important consequence and prediction of the EMBH model:

1. the most general GRB is composed of the P-GRB and and the afterglow (see Fig. 11). The relative intensity of the P-GRB and the E-APE is a function of the $B$ parameter.

2. for $B<3.5 \times 10^{-5}$ the energy of the P-GRB is larger then the one of the E-APE and the energy of the dyadosphere is mainly emitted in what have been called the "short bursts". Their afterglow have been systematically lower then the BATSE threshold.

3. for $B>3.5 \times 10^{-5}$ the energy of the E-APE predominates and the energy of the dyadosphere is mainly carried by the ABM pulse and emitted in the afterglow. The corresponding E-APE have been improperly called "long bursts". 


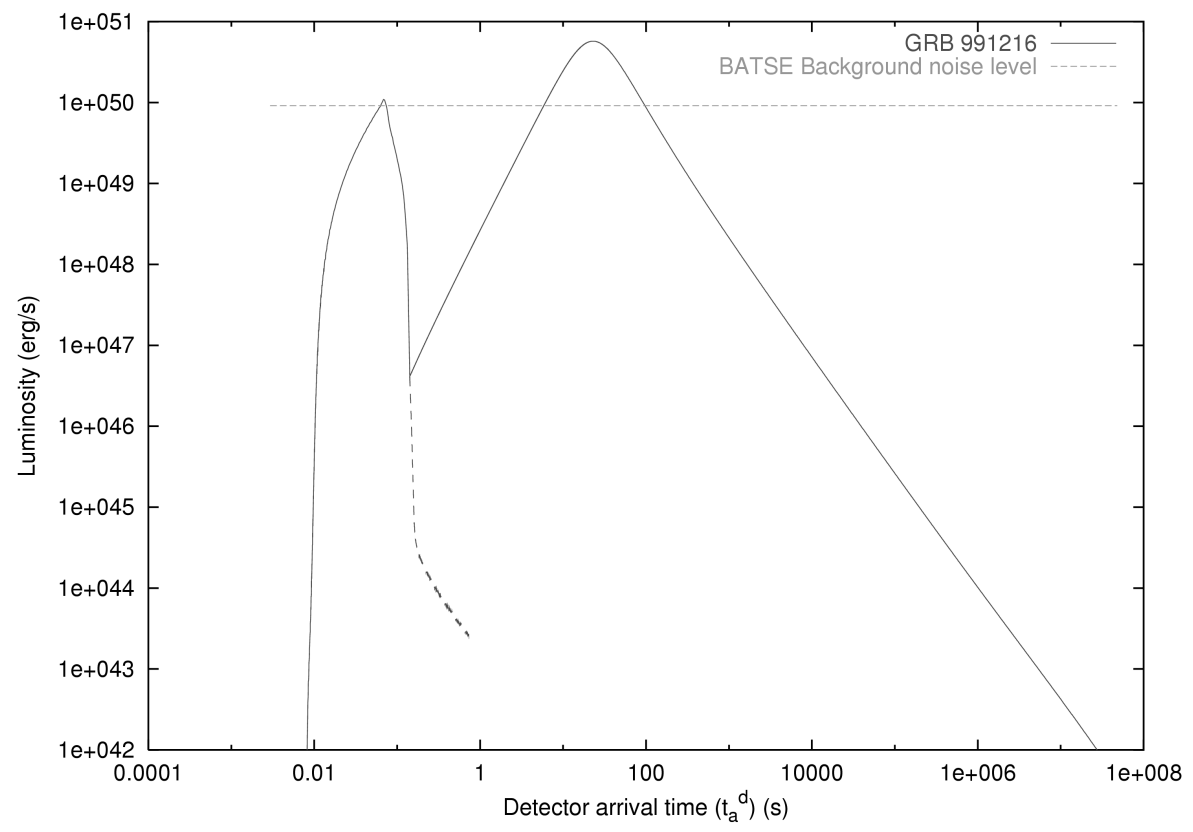

Fig. 11. Diagram showing the full picture of the model, with both P-GRB and E-APE and their relative intensities and time separation. The numerical values are presented and tabulated in [50].

It is interesting that this classification also explains at once the recently found conclusion that the distribution of short GRBs and long GRBs have essentially the same characteristic peak luminosity [56] and the fact that the short bursts are systematically harder then long bursts, see [50] for details.

Similarly the application of the RSTT and IBS paradigm has naturally conduced as to the concept of induced gravitational collapse 46], in order to explain the observed iron lines emission in the late phases of the afterglow of GRB 991216 and analogous effects in three additional GRBs showing clear correlation with supernovae. This is the topic of fortcoming publications [52].

The understanding of the role of P-GRB and E-APE in GRB 991216, the fact that both their absolute and relative intensities have been predicted within a few percent accuracy as well as that their arrival time has been computed with the precision of a few milliseconds, see [50], and Figs. 7,9,11, can be considered one of the major success of the EMBH theory.

New space missions have to be conceived to explore, on additional GRB sources, the theoretical predictions in the first $10^{2}$ seconds of Fig. 11. This region has been left vastly unexplored by the BATSE data due to the high threshold. Current missions are exploring with great accuracy mainly the later phases of the afterglow. These observations as well as the ones of short bursts, which in 
the EMBH theory are P-GRB emissions, are indeed crucial, since indeed we re-hiterate: all general relativistic and relativistic quantum field theory effects originating in the process of gravitational collapse during the formation of the EMBH are, in principle, encoded in the structure of the P-GRB [6,49,53].

The long lasting debate, started in Princeton in 1971, of how an EMBH is formed has also by now been clarified in [43]. The needed charge segregation process occurs in the magnetosphere of a rotating magnetized star. The charged collapsing core, surrounded by an oppositely charged remnant, approach the EMBH final stages in $\sim 30$ seconds for a $10 M_{\odot}$ progenitor star. The leading process of discharge of the EMBH is due to the vacuum polarization process in view of their very short time scale $10^{-19}$ seconds, see [37.

The EMBH theory can now be applied to all other GRB sources.

\section{References}

1. BATSE Rapid Burst Response, 1999, http://gammaray.msfc.nasa.gov/ kippen/batserbr

2. Bianco, C.L., Ruffini, R., Xue, S.-S., 2001, A\&A, 368, 377

3. Bianco, C.L., Chardonnet, P., Ruffini, R., Xue, S.-S., 2002, in preparation

4. Biretta, J.A., Sparks, W.B., Macchetto, F., 1999, ApJ, 520, 621

5. Bloom, J.S., Kulkarni, S.R., Djorgowski, S.G., 2000, submitted to Astron. Journ., astro-ph/0010176

6. Cherubini, C., Ruffini, R., Vitagliano, L., 2002, in preparation

7. Christodoulou, D., Ruffini, R., 1971, Phys. Rev. D, 4, 3552

8. Corbet, R., Smith, D.A., 2000, in "Rossi2000: Astrophysics with the Rossi X-ray

Timing Explorer", Greenbelt, USA

9. Costa, E., invited talk in "IX Marcel Grossmann Meeting on General Relativity", V. Gurzadyan, R. Jantzen and R. Ruffini Ed., World Scientific, 2001, in press

10. Damour, T., Ruffini, R., 1975, Phys. Rev. Lett., 35, 463

11. Dermer, C.D., Chiang, J., Böttcher, M., 1999, ApJ, 513, 656

12. Dermer, C.D., Mitman, K.E., 1999, ApJ, 513, L5

13. Dermer, C.D., 2000, astro-ph/0005440

14. Eichler, D., Livio, M., Piran, T., Schramm, D.N., 1989, Nature, 340, 126

15. Einstein, A., 1905, Ann. Phys. (Germany), 17, 891

16. Fenimore, E.E., 1999, ApJ, 518, 375

17. Fenimore, E.E., Cooper C., Ramirez-Ruiz, E., Sumner, M.C., Yoshida, A., Namiki, M., 1999, ApJ, 512, 683

18. Fryer, C.L., Woosley, S.E., Herant, M., Davies, M.B., 1999, ApJ, 520, 650

19. Giacconi, R., Ruffini, R., Ed. and coauthors, Physics and Astrophysics of Neutron Stars and Black Holes, North Holland, Amsterdam, 1978

20. Halpern, J.P., Uglesich, R., Mirabal, N., Kassin, S., Thorstensen, J., Keel, W.C., Diercks, A., Bloom, J.S., Harrison, F., Mattox, J., Eracleous, M., 2000, ApJ, 543, 697

21. Halzen, F., "High energy neutrino astronomy" in "Weak Interactions and Neutrinos, Proceedings of the 17th International Workship. Cape Town, South Africa", Edited by C. A. Dominguez and R. D. Viollier, World Scientific Publishers (Singapore, 2000), p.123

22. Heisenberg, W., Euler, H., 1935, Zeits. Phys., 98, 714

23. MacFadyen, A.I., Woosley, S.E., 1999, ApJ, 524, 262 
24. Mészáros, P., Rees, M.J., 1992a, MNRAS, 257, 29p

25. Mészáros, P., Rees, M.J., 1992b, ApJ, 397, 570

26. Mészáros, P., Rees, M.J., 1993, ApJ, 405, 278

27. Mészáros, P., Rees, M.J., 1997, ApJ, 482, L29

28. Mirabel, I.F., Rodriguez, L.F., 1999, A.R.A.A., 37, 409

29. Narayan, R., Paczyński, B., Piran, T., 1992, ApJ, 395, L83

30. Paczyński, B., 1991, Acta Astronomica, 41, 257

31. Paczyński, B., Xu, G., 1994, ApJ, 427, 708

32. Paczyński, B., 1998, ApJ, 494, L45

33. Panaitescu, A., Kumar, P., 2001, ApJ, 560, L49

34. Piran, T., talk at 2000 Texas Meeting, see also astro-ph/0104134

35. Piro, L., et al., 2000, Science, 290, 955

36. Preparata, G., Ruffini, R., Xue, S.-S., 1998, A\&A, 338, L87

37. Preparata, G., Ruffini, R., Xue, S.-S., 2002, in "Proceedings of The Seventh ICRA Network Workshop and The Seventh Korean-Italian Meeting on General Relativity". 38. Rees M.J., 1966, Nature 211, 468

39. Rees, M.J., Mészáros, P., 1994, ApJ, 430, L93

40. Ruffini, R., 1998, in "Black Holes and High Energy Astrophysics", Proceedings of the 49th Yamada Conference Ed. H. Sato and N. Sugiyama, Universal Ac. Press, Tokyo, 1998

41. Ruffini, R., Salmonson, J.D., Wilson, J.R., Xue, S.S., 1999, A\&A, 350, 334, A\&AS, 138,511

42. Ruffini, R., Salmonson, J.D., Wilson, J.R., Xue, S.S., 2000, A\&A, 359, 855

43. Ruffini R., 2002, in "Proceedings of the Ninth Marcel Grossmann Meeting on General Relativity", World Scientific, Singapore, in press

44. Ruffini, R., Bianco, C.L., Chardonnet, P., Fraschetti, F., Xue, S.-S., 2001, ApJ, 555, L107

45. Ruffini, R., Bianco, C.L., Chardonnet, P., Fraschetti, F., Xue, S.-S., 2001, ApJ, 555, L113

46. Ruffini, R., Bianco, C.L., Chardonnet, P., Fraschetti, F., Xue, S.-S., 2001, ApJ, $555, \mathrm{~L} 117$

47. Ruffini, R., Bianco, C.L., Chardonnet, P., Fraschetti, F., Xue, S.-S., 2001, Nuovo Cimento B, 116, 99

48. Ruffini, R., in "Fluctuating Paths and Fields - Dedicated to Hagen Kleinert on the Occasion of His 60th Birthday", Eds. W. Janke, A. Pelster, H.-J. Schmidt, and M. Bachmann, World Scientific, Singapore, 2001, p. 771

49. Ruffini, R., Vitagliano, L., 2002, submitted for pubblication

50. Ruffini, R., Bianco, C.L., Chardonnet, P., Fraschetti, F., Xue, S.-S., 2002, submitted for publication

51. Ruffini, R., Bianco, C.L., Chardonnet, P., Xue, S.-S., 2002, in preparation

52. Ruffini, R., Bianco, C.L., Chardonnet, P., Xue, S.-S., 2002, in preparation

53. Ruffini, R., Vitagliano, L., Xue, S.-S., 2002, in preparation

54. Salmonson, J.D., Wilson, J.R., Mathews, G.J, 2001, ApJ, 553, 471

55. Sari, R., Piran, T., 1997, ApJ, 485, 270

56. Schmidt, M., 2001, ApJ, 559, L79

57. Schwinger, J., 1951, Phys. Rev., 98, 714

58. Strong, I.B., in "Neutron Stars, Black Holes and Binary X-Ray Sources", Gursky, H. and Ruffini, R., editors, D. Reidel Publishing Company, 1975

59. Takeshima, T., Markwardt, C., Marshall, F., Giblin, T., Kippen, R.M., 1999, GCN Circ. 478 http://gcn.gsfc.nasa.gov/gcn/gcn3/478.gcn3 
60. Vietri, M., Stella, L., 1998, ApJ, 507, L45

61. Vietri, M., Stella, L., 1999, ApJ, 527, L43

62. Wilson, J.R., Mathews, G.J., Marronetti, P., 1996, Phys. Rev. D, 54, 1317

63. Woosley, S.E., 1993, ApJ, 405, 273 